\title{
Trends in Ontology of VANET
}

\author{
A. A. Obiniyi \\ Department of Mathematics \\ Ahmadu Bello University \\ Zaria, Nigeria.
}

\author{
S. K. Aina \\ Department of Mathematics \\ Ahmadu Bello University \\ Zaria, Nigeria.
}

\author{
M. B. Hammawa \\ Department of Mathematics \\ Ahmadu Bello University \\ Zaria, Nigeria.
}

\begin{abstract}
Ontology are widely used currently because of the ability to find solution to the problem of interoperateability between different domain of discourse, various language of usage to develop and query the ontology and several technology supported by ontology and semantic language. Eventhough a lot of research has been done on ontology application to Vehicular Adhoc Network (VANET) in aspect of Intelligent Transportation System (ITS). In this paper, a carefully review of already existing VANET systems that have employed the use of Semantic Web is carried out. And subsequently highlights some prospects in further exploitation of recent developments in the Semantic Web for the provisioning of novel research work.
\end{abstract}

\section{Keywords}

Intelligent Transportation System (ITS), Vehicular Adhoc Network (VANET) and Ontolgy.

\section{INTRODUCTION}

The concept of networking in computing became necessary even as information sharing among group of users, either on intra or inter relational basis, began to affect the communication pattern among people from all walks of life. Majorly, what took toll then was simple networking of computer to computer, popularly called point-to-point connection. Advances in research birthed several networking topologies of which they include ring, mesh, star, bus topologies and a host of others. And networking among devices has been categorized into local area network and wide area network. Technically, these networking patterns described above have been stationed. Stationed in the sense that, each participating node (machine) in the network does not move or displace itself from one network to another network even while it carries out its network related task, say file sharing. Hence, a network administrator configures the network with this notion in view. Furthermore, machines participating in networks were dynamically assigned internet protocol (IP) address. This made connectivity quite liberal, providing intending machines the privilege of attaching and detaching themselves to the network at any time. Hence, a node can enter and reentering a network several times and at the same time utilizing different IP addresses. But, since the manual IP configuration is seemingly left out of the hands of the network administrator, there arose the need for a dynamic host configuration protocol (DHCP) server which dynamically assigns IP address to machines attempting connectivity to the network and as well reclaiming the IP address of machines that are disconnecting themselves from the network.

Technological advances began to give new and better shape to networking. Mobile devices and other electronically driven machines having the tendency of been moved from one point to another even while they perform their functions began to demonstrate the need for network connectivity even while in motion. This necessitated more research that brought forth the concept of mobile ad-hoc network (MANET) and vehicular ad-hoc network (VANET). This network pattern allows host or nodes to connect and disconnect to and from the system respectively at any time, though they continue to carry out their network aided tasks while reconnecting to other network. The word ad-hoc intends to point out that each node in the network are not permanent but are coupled or network together to quickly and jointly perform a task afterward they will be disconnected. According to [1] and [2] Ad-hoc network is said to be an infrastructure less network with no fixed routers, hosts or wireless base stations. Vehicular Adhoc Networks (VANETs) represent a rapidly emerging, particularly challenging class of Mobile Ad Hoc Networks (MANETs).

\section{VEHICULAR AD HOC NETWORK (VANET)}

A vehicular ad hoc network (VANET) is an application of mobile network where each participating vehicle communicates with each other (peer to peer manner) and as well serve as a wireless router.

VANETs which are known to be a distributed, self-organizing communication networks built up by moving cars as nodes in a network to create a very high mobility of nodes and limited degrees of freedom in nodes movement patterns. It turns every participating car into a wireless router or node, allowing cars approximately 100 to 300 meters of each other to connect and in turn, create a network with a wide range.

VANETs are developed for enhancing the driving safety and comfort of automotive users. The VANETs can provide wide variety of service such as Intelligent Transportation System (ITS), Intelligent Vehicular ad hoc network (InVANET), Dedicated short-range communication (DSRC), Roadside-tovehicle communication (RVC) e.g. safety applications[3].

VANETs has gained an important part of the interest of researchers and become very popular. More specifically, VANETs can operate without fixed infrastructure and can survive rapid changes in the network topology [2].

\section{ONTOLOGY}

Philosophically, ontology is the study of the kinds of things that exist. It is usually believe that ontologies "carve the world at its joints." Ontologies [4], which are used in order to support interoperability and common understanding between the different parties, are a key component in solving the problem of semantic heterogeneity, thus enabling semantic interoperability between different web applications and services.

Ontologies provide a common understanding of a domain that can be communicated between people of heterogeneous and widely spread application systems. Ontology has been used in several domain of computer science even in Ad hoc network system to find solution, such as to vulnerability in security aspect [5]. 
Ontology provides more clarification and simplicity to domain of discourse. It makes understanding easy between applications and people of either same or different domain.

\section{A REVIEW OF SOME SEMANTIC WEB BASED VANET SYSTEMS}

There have been several studies on ontology based VANET. For example in [6] an ontology modelling approach for assisting vehicle drivers through safety warning messages during time critical situation was proposed. The work aims at creating alert messages based on the context aware parameters using driving situation and such as driver activity and environment. The work was implemented using ontology modelling and XML format to manage the I-DAS (intelligence- driver assistance system) parameter for the illustration in the update /maintenance in the work. Java Framework was used to generate safety alert in several driving situations. The future work was to consider a more comprehensive extension of the overtaking knowledge base supposed to take into consideration the decision of a human driver during traffic with reasoning process into account.

The work [7], a high-level representation of automated vehicle, other vehicles and their environment was proposed which can help drivers in taking such "illegal" but practical relaxation decisions. Implementation of the work was carried out using high-level representation which uses ontology in topological knowledge and inference rules in other to make decision on the next high-level motion of an automated vehicle in order to support a driver. Two instances were given in the work.

a. An example of a situation where vehicle was stuck on the road because of a vehicle ahead of it stop on the lane with no good reason that might take a longer time such unloading things, meanwhile according to the traffic regulation the vehicle cannot overtake.

b. An example of a circumstance on reaching a roundabout, a car ahead of a driver stopped on the lane with an engine problem and the with traffic regulation the driver has to wait behind the defective until the car continues its movement again, which could take a longer hour to rectify.

Human drivers can manage with the two situation $a$ and $b$ by checking the oncoming vehicle behind it and move on but in the case of autonomous vehicle driven by a computer that is intelligent robotic vehicle in the two instance $a$ and $b$ since the vehicle is considering the traffic regulation the vehicle will continue waiting (trapped) till the vehicle ahead it continues it movement again. In these situations for a cycab to reasoning like a human driver (imitate) a decision needed to be take that is should a cycab follow its obstacle avoidance algorithm, then change lane or should it follow traffic regulation that is stay on its lane.

The works focus mainly on the internal part of each vehicle only specifically its decisional part, meanwhile environmental scenario is also very important.

In [8] discussed about embed a symbolic representation that is ontology in a component of each vehicle in order for it to deals with emergency situation and also to manage the situation at intersection in order to reasoning on its usage of traffic rules. It also discusses about the automation level of the vehicle such that it will observed the state of a driver from fully aware through drowsiness to full asleep by using a camera detecting eye opening level, blink frequency and blink duration. They later proposed to determine the maximum autonomy level that a vehicle can be adopt in order to cope with the current state of the environment so that it can ensure a safe driving. Automation means all the modes which imply actions were done by the system through the actuators, including actions against the driver's intention such as emergency braking. The work did not cover providing advice, warning to the driver and like blind spot detection is not considered as an automation mode.

Ontology was used to model VANETS security in terms of identifying intrusion based on semantics [9]. The work has shown different techniques of ontology-based intrusion detection, summary of ontology web language and how ontology can be used to create namespaces and declaration of class in order to identify the relationship between the parameter of vulnerabilities in term of intrusion detection. This work was mainly on use of ontology to solve intrusion detection for vehicular ad hoc networks. The work did not reflect on other aspect of ontology of VANET and their environment.

An ontology-based framework was proposed which provides human like reasoning about the driving environment from the facts gathered from on-board sensor, maps and vehicle state [10]. This entails the information from different parameters available in road situations. The work uses terminological box (TBox) and an assertional box (ABox) to describe the concepts of their ontology in term of class and relationships between classes such as object properties and their rules. Also declare an instance of concepts such as individuals. The work used OWL to edit and verify the ontology in protégé and swoops. According to the work the objective was not to design an exhaustive ontology that considers every type of context entity, but rather to design a coherent, easily extendable ontology based framework and it can only reason on contexts compatible with it, that is contexts which only meet entities which have been defined in the TBox. This means that for an intensive use of the ontology, it has to be extended to take new types of entities into consideration. The work primarily based on ADAS (Advance driving Assistance System) was concerning the interaction between the lead vehicle, the pedestrian and the road intersection. In our current work we aim at developing a dynamically enrich knowledge base which will extend the issue of lead vehicle considering ADAS and as well as given a suggestion on road usage for a human driver.

\section{FUTURE OF SEMANTIC WEB BASED VANET SYSTEMS}

Some points that can be highlighted and discussed here include:

a. That high level of human-nature intelligence could be deployed into VANET system when semantic web rule language is combined with non-semantic rule languages in artificial intelligence.

b. We see a VANET system that extends to enclose the pedestrian system into it structure. This argument is based on the fact that there are already research works that have successfully being deployed in management and automating the pedestrian system, hence, an ontologically modeled knowledge base modularized into their categories (pedestrian and traffic system) will serve as a booster to this goal.

c. VANET systems do not have a car tracking system. Apart from congestion management and control, a major vehicular-road traffic tasks that is cogent to 
such system is the car/vehicular tracking system. As stated in the second point, when a knowledge base of all vehicle in the system are sustained, then a semantic web based tracking agent can be easily plugged into the VANET system.

d. Future VANET systems will greatly demonstrate a more fine-grained level of information sharing among its nodes. This will be made possible as more powerful constructs are being added to the existing ones that constitute the rule and query languages that abound in semantic web.

\section{CONCLUSION}

This review work focused on identifying research works that had being successfully done in the field of vehicular ad-hoc network; specifically in exploiting the use of semantic web vision. Particularly, this research made reference to the strengths inherent in these systems. However, it must be noted here that there remains much to be harnessed in the semantic web train in entrenching intelligence into the VANET system.

Ambient computing is a branch of AI that is being borne on the wings of the Semantic web vision/technology. It promotes the incorporation of machine intelligence into the human environment. Now, seeing the VANET system as an environment that man interacts with, this research wishes to note here that a high level of autonomous vehicular system may be realized in VANET. For example, one could imagine a self-tuning feature added to both the vehicles and traffic systems that comprises of the VANET system. Self-tuning in the sense that the components of VANET, (vehicular objects for example) are able to intelligent change the course movement or direction given a just arising situation or data input.

Secondly, we observe that data representation limitations are greatly submerged in the semantic web. Hence, a more refined and expert modelled ontology or knowledge base for the VANET system is a way of achieving a finer grain of intelligence. Meanwhile, a combination of rule languages and reasoners will further enhance entailments in the VANET system.

\section{REFERENCES}

[1] Tarik, T; Ehssan, S. and Abbas. J. (2007): "A Stable Routing Protocol to Support ITS Services in VANET Networks", IEEE Transactions on Vehicular Technology, 56(6): 3337- 3347.

[2] Shastri, A; Dadhich, R. and Poonia R. C. (2011): Performance Analysis of On-Demand Routing Protocols for Vehicular ad-hoc Networks. International Journal of Wireless \& Mobile Networks (IJWMN) Vol. 3, No. 4, pp $103-111$.

[3] Kirthiga, N. and Karthik S. (2014): Vehicular Traffic ReRouting For Avoid the Traffic Congestion in VANET. International Conference on Engineering Technology and Science-(ICETS'14) On 10th \& 11th February Organized by Department of Civil, CSE, ECE, EEE, Mechnical Engg. and S\&H of Muthayammal College of Engineering, Rasipuram, Tamilnadu, India www.ijirset.com $p p 1530-1533$.

[4] Mihoubi, H; Simonet, A. and Simonet, M. (2000): "An Ontology Driven Approach to Ontology Translation", In Proceedings of DEXA, pp.573-582.

[5] Verma, A. and Gujral, M. S. (2012): An Ontological View of Trusted OLSR Protocol of Ad hoc Network. Special Issue of International Journal of Computer Applications on Issues and Challenges in Networking, Intelligence and Computing Technologies - ICNICT 2012. (0975-8887) Pp $18-21$.

[6] Saravanan, K; Arunkumar, T. and RameshBuba, K. (2010): An Intelligent Driver Assistance System (I-DAS) for Vehicle Safety Modelling using Ontology Approach International Journal of UbiComp (IJU), Vol.1, No.3, page $15-29$.

[7] Morignot, P. and Nashashibi, F. (2012): An OntologyBased Approach to relax Traffic Regulation for Autonomous Vehicle Assistance. INRIA Rocquencourt, Team IMARA, Domaine de Voluceau, B.P. 105, 78153 Le Chesnay, France. Pp $1-8$.

[8] Pollard, E; Morignot, P. and Nashashibi, F. (2013): An Ontology-based Model to Determine the Automation Level of an Automated Vehicle for Co-Driving. Published in16th International Conference on Information Fusion. hal-00838680, version 1 - 27.

[9] Erritali, M; EL-Ouahidi, B; Hssina, B; Bouikhalene, B; and Merbouha, A. (2013): An Ontology-Based Intrusion Detection for Vehicular Ad Hoc Networks: Journal of Theoretical and Applied Information Technology 31st July 2013. Vol. 53 No.3, pp $410-414$.

[10] Armand, A; Filliat, D. and Ibañez-Guzman, J. (2014): Ontology-Based Context Awareness for Driving Assistance Systems. Intelligent Vehicles Symposium Proceedings, 2014 IEEE Dearborn, MI. 8-11 June 2014 14452153: Pp 227 - 233. 